\title{
Long term prognosis of recurrent haematuria
}

\author{
P F W MILLER, N I SPEIRS, S R APARICIO, M LENDON, J M SAVAGE, \\ R J POSTLETHWAITE, J T BROCKLEBANK, I B HOUSTON, AND S R MEADOW
}

Department of Paediatrics and Child Health, St James's University Hospital, Leeds and Royal Manchester Children's Hospital

SUMmaRY A long term follow up study of 100 children referred with recurrent haematuria for at least one year to two regional paediatric nephrology units is described. The mean duration of follow up was $8 \cdot 2$ years. An adequate renal biopsy was obtained in 96 and eight cases of Alport's syndrome and 10 of IgA nephropathy were diagnosed $(20 \%$ and $26 \%$ respectively of the biopsies examined by electron microscopy and immunofluorescence).

Five patients developed end stage renal failure and six hypertension requiring treatment, with the occurrence of these complications increasing progressively with increasing duration of follow up ( $1 \%$ at five years compared with $12 \%$ at 10 years). Adverse prognostic features were persistence of microscopic haematuria, proteinuria at presentation, and appreciable changes on renal biopsy. Eighty four patients had first degree relatives tested for haematuria; $30 \%$ of these families had another affected member. With long term follow up recurrent haematuria is associated with considerable morbidity and potential mortality.

Children presenting with what is generally called recurrent haematuria or benign haematuria have several different underlying diseases. The terms are somewhat inappropriate as many of the patients have persistent microscopic haematuria between episodes of intermittent macroscopic haematuria and also because some develop serious complications. Although there have been several clinical and pathological studies that have emphasised the benign prognosis; ${ }^{1-7}$ their follow up was short and most biopsies were performed before the use of electron microscopy and immunofluorescence. We have studied a large series of children to determine the long term prognosis, factors that may help to predict prognosis, and the incidence of the different diseases underlying this syndrome.

\section{Methods}

We studied 100 consecutive patients who had had frank or microscopic haematuria for at least one year. They were referred by regional paediatricians and local general practitioners between 1967 and 1978 to supraregional paediatric nephrology clinics in Leeds and Manchester. Patients were included if at onset they were normotensive, did not have oedema, and had normal serum creatinine and $\mathrm{C}_{3}$ complement values and sterile urine cultures.
Patients known to have a family history of Alport's syndrome or renal failure were excluded. Most had red or white cell casts on urine microscopy and all had normal intravenous urograms. Measurements of height, weight, and blood pressure were made; urine was tested for blood and protein; and a percutaneous renal biopsy was performed in all cases. Audiograms were performed initially on patients suspected of having Alport's syndrome, and more recently on all patients. Ninety seven specimens adequate for light microscopy were obtained. Many of the patients had their biopsy before the routine use of electron microscopy and immunofluorescent staining. All of the biopsies have been jointly reviewed recently by two pathologists (SA and ML) to classify them by light microscopy into three groups:

Group 1. Normal or minor changes, showed either nil disease, mild mesangial hypercellularity or mild mesangial thickening, or both, in up to $20 \%$ of glomeruli; capsular adhesions in up to $20 \%$ of glomeruli; occasional global glomerular obsolescence without tubular atrophy or one focus of tubular atrophy without glomerular sclerosis.

Group 2. Focal nephritis, showed segmental mesangial hypercellularity or matrix thickening, or both, in up to $80 \%$ of glomeruli; capsular adhesions in up to $80 \%$ of glomeruli; epithelial crescents; 
segmental or global glomerular sclerosis; more than one focus of tubular atrophy; focal capillary wall thickening; focal interstitial inflammatory infiltrates.

Group 3. Diffuse nephritis, showed segmental or global proliferative changes affecting more than $80 \%$ of glomeruli.

Where appropriate, the diagnoses of $\operatorname{IgA}$ nephropathy and Alport's syndrome were also made. IgA nephropathy was diagnosed where immunofluorescence showed IgA to be the predominant immunoglobulin detected. Alport's syndrome was diagnosed if there was haematuria and at least two of the following:

(i) Typical widespread glomerular basement membrane splitting and multilayering.

(ii) Sensorineural hearing loss in the patient.

(iii) A first degree relative with sensorineural hearing loss. (Our usual criteria would also include those with a family history of renal failure, though such patients have already been excluded from this study).

Ninety seven of the 100 children studied at presentation have been followed up within the past year, the other three patients who refused full follow up consider themselves healthy and are leading normal lives. The 97 patients have all been examined more than five years after their presentation and have had checks on general health, growth, and blood pressure; urine testing for blood and protein; and plasma creatinine values determined. Approximately half of the patients had been discharged from follow up having received a good prognosis or had outgrown the paediatric clinics. We also tested as many first degree relatives as we were able to contact, all of whom considered themselves to be healthy.

\section{Results}

Clinical features at onset. Presenting symptoms were dark urine only in 55, dark urine and systemic symptoms in 30 (abdominal pain, arthralgia, or general malaise), and microscopic haematuria as an incidental finding in 15 . The age of onset was 5 months to 14 years (mean 7.2 years) with the incidence steadily increasing to a peak at 10 years and falling away thereafter. There was noticeable male predominance $(3 \cdot 25: 1)$ which was present in each age group. During the study it became increasingly clear that the patients had consistent patterns of haematuria. These were intermittent macroscopic haematuria with persistent microscopic haematuria in 56, intermittent macroscopic haematuria with clearing between attacks in 29 , and persistent microscopic haematuria in 15 . The latter group was included as in many ways it resembled the other two groups, particularly the group with intermittent macroscopic and persistent microscopic haematuria. In 68 patients macroscopic haematuria was precipitated by recognisable factors-exercise in 10 , upper respiratory tract infection in 47 , and either in 11. Severe proteinuria was present in 33 patients at presentation; $27(82 \%)$ of these had intermittent macroscopic with persistent microscopic haematuria, three $(9 \%)$ had intermittent macroscopic haematuria and three $(9 \%)$ had persistent microscopic haematuria.

Renal biopsy findings. An adequate biopsy for light microscopy was obtained in 96 cases, for immunofluorescence in 35 , and for electron microscopy in 39 . By light microscopy, 36 had normal or minor changes, 55 focal nephritis, and five diffuse proliferative nephritis. Immunofluorescent staining was diagnostic of $\operatorname{IgA}$ nephropathy in 10 cases $(26 \%$ of those examined by this technique): four of these patients had minor changes and six had focal nephritis on light microscopy. Eight patients were diagnosed as having Alport's syndrome by combined pathological and clinical criteria; six of these had typical widespread basement membrane changes, ( $15 \%$ of biopsies studied by electron microscopy), one had focal areas of basement membrane suggestive of Alport's syndrome and associated with bilateral sensorineural hearing loss. One patient did not have electron microscopy performed but had sensorineural hearing loss, a family history of sensorineural hearing loss and nephritis, and macrothrombocytopathia. Platelet abnormalities are associated with Alport's or an Alport-like syndrome. $^{8}$ Another four children had basement membrane changes typical or suggestive of Alport's syndrome but no other suggestive features. Any of these children may be firmly diagnosed as having Alport's syndrome in the future.

\section{Follow up studies}

\section{Clinical findings at the end of follow up}

Sixty three patients still had haematuria and 11 had developed serious complications-end stage renal failure in five and hypertension requiring treatment in six, one of whom had a raised serum creatinine concentration of $160 \mu \mathrm{mol} / \mathrm{l}$. The breakdown of complications is shown in Fig. 1. Seventeen patients developed or had increasing proteinuria during the study, but in 10 this cleared or reduced. The complications became increasingly frequent with increasing duration of follow up (Fig. 2). Only one $(1 \%)$ of the 100 patients seen five years after the onset of haematuria had hypertension, whereas five 


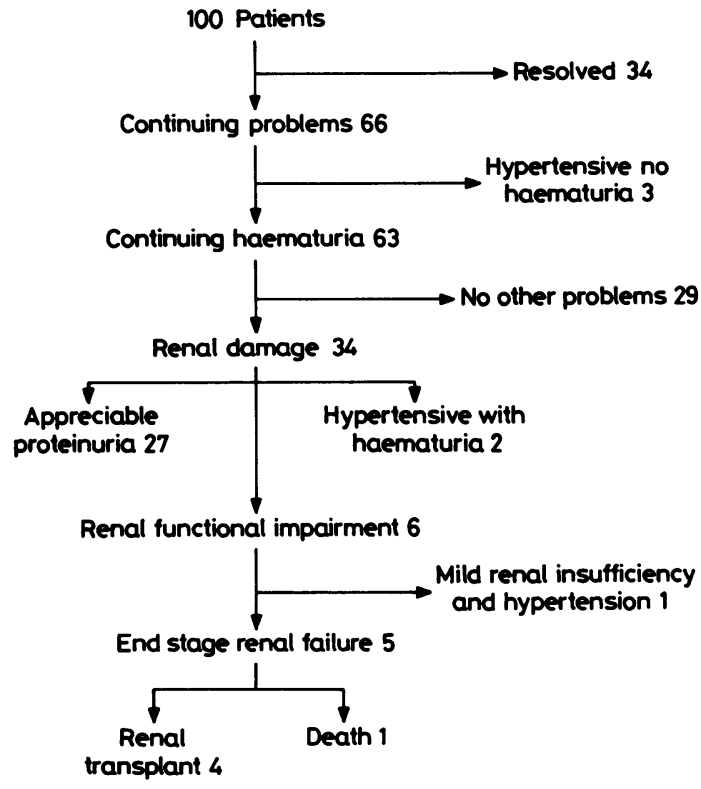

Fig. 1 Clinical outcome in 100 patients with recurrent haematuria.

$(12 \%)$ of the 43 patients followed up for 10 years had developed hypertension or renal failure. Of the 100 patients studied, in only 37 had their haematuria resolved; 63 continued to have haematuria for more than five years.

Apart from the children with renal failure, growth was normal and blood pressure for age was normally distributed except for those noted to be hypertensive. Audiometry was performed in those suspected

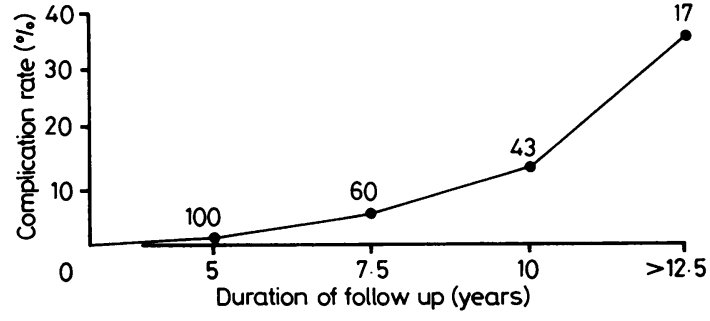

Fig. 2 Prevalence of complications (hypertension or chronic renal failure) related to duration of follow up.

Figures indicate the number of patients followed up at each time interval.

of having Alport's syndrome, and more recently in all patients with recurrent benign haematuria. Twelve of the 48 patients tested had sensorineural hearing loss, seven of whom had Alport's syndrome.

An appreciable degree of hearing loss was found in 12 patients, seven of whom have Alport's syndrome. In five cases this hearing loss was detectable, either clinically or on audiometry, at the time of presentation. Ten patients now have a degree of hearing loss that is severe enough to require a hearing aid.

\section{Analysis of factors which might help to predict prognosis (Table 1)}

Renal biopsy findings. No patients with a normal biopsy or minor changes developed renal failure or hypertension, all who did exhibited considerable changes in biopsy findings (Table 2). None of the patients with a type I biopsy and IgA nephropathy or Alport's syndrome has developed complications, whereas three with type II or type III biopsies

Table 1 Factors affecting the prognosis of recurrent haematuria

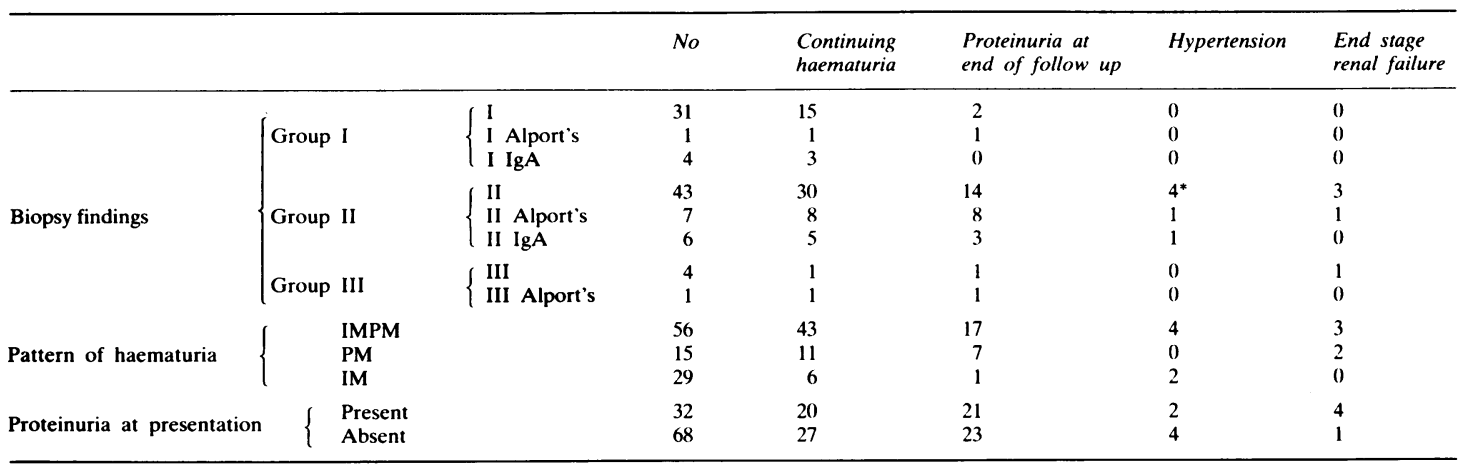

*One patient had hypertension and a slightly raised serum creatinine value $(160 \mathrm{umol} / \mathrm{l})$.

For biopsy classification see text.

IMPM=intermittent macroscopic persistent microscopic haematuria; PM=persistent microscopic hacmaturia; IM=intermittent macroscopic haematuria. 
Table 2 Summary of patients who developed complications

\begin{tabular}{|c|c|c|c|c|c|c|c|}
\hline $\begin{array}{l}\text { Case } \\
\text { no }\end{array}$ & $\begin{array}{l}\text { Pattern of } \\
\text { haematuria }\end{array}$ & Complication & $\begin{array}{l}\text { Time to develop } \\
\text { complication (yrs) }\end{array}$ & $\begin{array}{l}\text { Biopsy } \\
\text { category }\end{array}$ & Biopsy diagnosis & $\begin{array}{l}\text { Electron } \\
\text { microscopy }\end{array}$ & $\begin{array}{l}\text { Immuno- } \\
\text { staining }\end{array}$ \\
\hline 1 & IMPM & Renal failure & 13 & III & $\begin{array}{l}\text { Diffuse proliferative } \\
\text { glomerulosclerosis }\end{array}$ & ND & $C_{3} \operatorname{IgM}$ \\
\hline $2^{*}$ & PM & Renal failure & 14 & II & Alport's syndrome & ND & $-v e$ \\
\hline 3 & PM & Renal failure & 10 & II & Focal segmental glomerulosclerosis & ND & ND \\
\hline 4 & IMPM & Renal failure & 13 & II & Focal segmental nephritis & ND & $-v e \lg A$ \\
\hline 5 & IMPM & Renal failure & 16 & II & Focal segmental glomerulosclerosis & ND & ND \\
\hline 6 & IMPM & Hypertension & 4 & II & Focal segmental glomerulosclerosis & -ve & ND \\
\hline 7 & IMPM & Hypertension & 6 & II & Alport's syndrome & Diagnostic & ND \\
\hline 8 & IM & Hypertension & 8 & II & $\operatorname{IgA}$ nephropathy & ND & $\operatorname{Ig} A$ \\
\hline 9 & IMPM & Hypertension $\dagger$ & 14 & II & Focal nephritis & ND & $-v e$ \\
\hline 10 & IMPM & Hypertension & 8 & II & Focal nephritis & ND & ND \\
\hline 11 & IM & Hypertension & 13 & II & Focal nephritis & ND & ND \\
\hline
\end{tabular}

*Patient diagnosed as having Alport's syndrome on clinical criteria.

tRepresents the patient with hypertension and raised serum creatinine value (160 umol/l).

$\mathrm{ND}=$ not done; IMPM =intermittent macroscopic persistent microscopic haematuria; $\mathrm{PM}=$ persistent microscopic haematuria: $\mathrm{IM}=$ intermittent macroscopic haematuria.

associated with these conditions did. This may be because the biopsy was performed at an earlier stage in the disease and complications may develop with time. Type II and type III biopsies were associated with a higher incidence of continuing haematuria; $52 \%$ of patients with type I compared with $70 \%$ of patients with type II or III biopsies had haematuria at the end of follow up. Type II and III biopsies were also associated with a higher incidence of proteinuria at the end of follow up; this was found in $8 \%$ of patients with type I biopsies compared with $34 \%$ of patients with type II or III biopsies. Another important finding concerns the four patients who developed complications associated with focal segmental glomerulosclerosis, they represent $36 \%$ of the patients who deteriorated. Three of the patients who developed complications (cases 9,10 , and 11 in Table 2) did not have firm clinicopathological diagnoses, though it should be noted that none of these patients had all three modalities of biopsy examination. These differences in prognosis between the histological groups are not explained by differences in duration of follow up as they were followed up for a similar duration.

Pattern of haematuria. Patients with each of the three disease patterns developed complications. Only those with persistent microscopic haematuria with or without intermittent macroscopic haematuria, however, progressed to end stage renal failure. The prognosis for these patients was also worse in other respects: $76 \%$ and $73 \%$ of patients with intermittent macroscopic and persistent microscopic haematuria and persistent microscopic haematuria respectively continued to have haematuria (haematuria detected in the previous year) compared with $20 \%$ with i termittent macroscopic haematuria. Similarly, $30 \%$ and $46 \%$ of patients with these first two patterns of haematuria respectively had proteinuria at the end of follow up compared with $3 \%$ of patients with intermittent macroscopic haematuria. Patients with intermittent macroscopic haematuria were less likely to develop renal failure or to have continuing haematuria or proteinuria at the end of follow up. Intermittent macroscopic haematuria seems to be associated with a better prognosis than persistent microscopic haematuria with or without intermittent macroscopic haematuria. The differences in prognosis are not due to differences in duration of follow up as these were similar.

Proteinuria detected at presentation. Four of the five patients who developed end stage renal failure had proteinuria detected at presentation, thougin a similar proportion $(6 \%)$ in the three groups developed hypertension. Proteinuria at presentation was also associated with a higher incidence of continuing haematuria $(60 \%$ compared with $40 \%$ who did not have proteinuria). Similarly, as expected, patients with proteinuria at presentation had a higher incidence of proteinuria at the end of follow up $(64 \%$ compared with $34 \%$ ). Patients who had proteinuria at presentation had a worse outcome-they were more likely to develop renal failure and have continuing haematuria and proteinuria at the end of follow up. This difference could not be explained by difference in the duration of follow up.

Family testing. Urine tests were performed on 274 first degree relatives of 84 patients. Twenty five of these families had at least one other affected member. Affected members of two generations were often found. The prognosis was not related to the presence of an affected family member; there was no particular pattern of haematuria in the index 
cases, nor was there a particular histopathological picture. No child with IgA nephropathy had an affected relative and two of the eight patients with Alport's syndrome did not have an identified affected relative.

Precipitating factors. Prognosis was not related to the presence or absence of precipitating factors in the attacks of macroscopic haematuria. All the patients with IgA nephropathy and half of the patients with Alport's syndrome had precipitating factors in some of their episodes of haematuria. Precipitating factors occurred in intermittent macroscopic haematuria with or without microscopic haematuria and with each histological category.

Associated symptoms. Prognosis was not related to the presence of associated symptoms. IgA nephropathy was particularly associated with systemic symptoms in six of 10 patients affected. These were abdominal pain in five, general malaise in three, and vomiting in two. Each pattern of haematuria and each biopsy category had patients with systemic symptoms.

In view of the small numbers of patients developing complications none of the prognostic factors tested achieves statistical significance by $\chi^{2}$ testing, with $\mathbf{P}=0 \cdot 05$. This means that the null hypothesis is not excluded, that is all of these differences may be due to chance.

\section{Discussion}

The prognosis for this group of children was considerably worse than in other reported series, ${ }^{1-7}$ and worse than most paediatricians would expect. There are several possible explanations: the duration and completeness of follow up, the inclusion of patients only if they had had haematuria for more than one year, or our referral pattern. The duration of follow up is clearly important as shown by Fig. 2 with increasing morbidity and potential mortality after five years. This morbidity is likely to increase still further in several adolescent boys with Alport's syndrome and increasing proteinuria who are likely to develop renal failure. Many of the patients were not being followed up by us, they had either been discharged with a good prognosis or referred to adult nephrologists who did not necessarily inform their paediatric colleagues of any complications. These organisational problems may lead to an over optimistic impression of the condition. We included only patients who had had haematuria for more than one year because we felt that they were most likely to have appreciable morbidity and to warrant a renal biopsy. The children were referred to us and were not detected by population screening as in some other studies. ${ }^{9}{ }^{10}$ Screening is likely to find a more mildly affected group. The population served by our two centres is approximately 8.5 million so the referring doctors will not have referred all of their patients with haematuria, only those they were most concerned about.

The factors associated with a poorer prognosis were severe changes on renal biopsy, persistent microscopic haematuria with or without intermittent macroscopic haematuria, and proteinuria at presentation to the nephrology centre. A normal biopsy or minor histological changes were associated with a good prognosis. Three modality examination of the biopsy would seem to be mandatory in order to obtain the maximal amount of diagnostic information. Only three patients who developed complications did not have a firm clinicopathological diagnosis and none of these had all biopsy modalities examined. A surprising feature of the review of the histopathological findings was the number of cases with the appropriate study who had IgA nephropathy or Alport's syndrome. Whether biopsies were examined by electron microscopy or immunofluorescence depended upon whether the biopsy was undertaken after these techniques became available.

Alport's syndrome is difficult to diagnose, especially without electron microscopy, in view of the variable timing and severity of the associated features. Alport described the association of familial nephritis and deafness ${ }^{11}$ and we have used this with the more recently described basement membrane changes as the basis of our diagnostic criteria.

Persistent haematuria is likely to be associated with a continuing pathological process, whereas intermittent haematuria may be associated with an intermittent insult with periods of repair in between. Alternatively, persistent haematuria may represent more serious disease since all those with Alport's syndrome had persistent microscopic haematuria with or without intermittent macroscopic haematuria. The poorer prognosis of children with microscopic haematuria may be partly due to its association with Alport's syndrome. Proteinuria at onset was also associated with a poorer prognosis.

Benign familial haematuria is a term used to describe a dominantly inherited condition associated with haematuria with or without proteinuria often associated with thinning of the glomerular basement membrane and a good prognosis. ${ }^{12-14}$ Some patients in this study had affected first degree relatives both with and without proteinuria, with various patterns of haematuria, and changes on renal biopsy; some of whom developed complications. Both Habib ${ }^{15}$ andDische ${ }^{16}$ have suggested that renal impairment may occur in patients showing thinning of the glomerular 
basement membrane. We feel that benign familial haematuria is likely to represent a group of different inherited diseases as has been suggested by Doherty et al. ${ }^{17}$

Severe clinical deterioration remains difficult to predict, particularly if someone with entirely normal renal function and absence of proteinuria for many years subsequently progresses, often rapidly, to end stage renal failure. We suggest that all children with this syndrome should have regular long term follow up with a check on blood pressure and renal function to detect complications and enable treatment to be given as early as possible. We advocate renal biopsy for patients who have had unexplained recurrent haematuria for more than a year for prognostic information, particularly when persistent microscopic haematuria is present and when proteinuria is detected at presentation.

It is interesting to speculate how many adult patients presenting with renal failure with changes suggestive of chronic glomerulonephritis may have had this syndrome associated with microscopic haematuria in childhood.

We thank Mrs W A Pearson for secretarial help and Drs M Mason and $G$ Scott for assistance with histological work.

\section{References}

1 Glasgow EF, Moncrieff MW, White RHR. Symptomless haematuria in childhood. $\mathrm{Br}$ Med $J$ 1970;ii:687-92.

2 Arneil GC, Lam CN, McDonald AM, McDonald M. Recurrent haematuria in 17 children. $B r$ Med J 1969;ii:233.

3 Ayoub EM, Vernier RL. Benign recurrent haematuria. Am J Dis Child 1965;109:217-23.

4 Johnston C, Shuler S. Recurrent haematuria in childhood. A five year follow up. Arch Dis Child 1969;44:483-6. s Schoeneman MJ, Earon Y, Spitzer A, Greifer I. Idiopathic persistent microscopic haematuria in children. Prognostic features. NY State J Med 1979;79:1714-8.

${ }^{6}$ Pardo V, Berion MG, Levi DF, Strauss J. Benign primary haematuria. Clinicopathologic study of 65 patients. Am J Med 1979;67:817-22.

${ }^{7}$ Bodian M, Black JA, Kobayashi N, Lake BD, Shuler SE. Recurrent haematuria in childhood. $Q J$ Med 1965;34:359-82.

${ }^{8}$ Bernheim J, Dechevanne M, Byron PA, et al. Thrombocytopenia macrothrombocytopathia, nephritis and deafness. Am J Med 1976;61:145-50.

9 Dodge WG, West EF, Smith EH, Bunce H. Proteinuria and haematuria in school children: epidemiology and early natural history. J Pediatr 1976;88:327-47.

10 Vehaskari VM, Rapola J, Koskimies O, Savilahti E, Vilska J, Hallman N. Microscopic haematuria in school children. Epidemiology and clinicopathologic evaluation. $J$ Pediatr 1979;95:676-84.

$"$ Alport AC. Hereditary familial congenital haemorrhagic nephritis. Br Med J 1927;i:504-6.

12 McConville JM, West CD, McAdams AJ. Familial and non familial benign haematuria. $J$ Pediatr 1966;69:207-14.

13 Piel CF, Biava CG, Goodman JR. Glomerular basement membrane attenuation in familial nephritis and 'benign' haematuria. J Pediatr 1982;101:358-65.

14 Yoshikawa N, White RHR, Cameron AH. Familial haematuria: clinico pathological correlation. Clin Nephrol 1982;17:172-82.

15 Habib R, Gubler M, Hinglais N, et al. Alport's syndrome: experience at Hospital Necker. Kidney Int 1982;21(suppl 11): 520-8.

16 Dische FE, Weston MJ, Parsons V. Abnormally thin glomerular basement membranes associated with haematuria, proteinuria or renal failure in adults. Am J Nephrol 1985;(in press).

${ }^{17}$ Doherty CC, Middleton DT, Hill CM. Hereditary glomerulonephritis of non-Alport type. Proc Eur Dial Transplant Assoc 1983;19:575-81.

Correspondence to Professor S R Meadow, Department of Paediatrics and Child Health, St James's University Hospital, Leeds LS9 7TF.

Received 8 January 1985 